\title{
X-RAY RESONANT MAGNETIC SCATTERING: APPLICATION TO THIN FILMS AND MULTILAYERS
}

\author{
D. RAOUX ${ }^{a}$, N. JAOUEN ${ }^{a}$, J.M. TONNERRE ${ }^{a}$ AND E. BONTEMPI ${ }^{b}$ \\ ${ }^{a}$ Laboratoire de Cristallographie, CNRS and UJF \\ B.P. 166, 38042 Grenoble Cedex 09, France \\ ${ }^{b}$ Laboratorio di Strutturistica Chimica, Universitá di Brescia \\ Via Branze 38, 25123 Brescia, Italy
}

\begin{abstract}
This paper reports on the use of a new technique to investigate the magnetic properties of thin films, multilayers and artificial structures, the X-ray resonant magnetic scattering at small values of the scattering vector. It can be used either by registering the reflectivity pattern or in a diffraction mode. In comparison with magneto-optical Kerr effect or neutron scattering, it offers an atomic selectivity due to the resonant excitation of a core electron, and even an electronic shell one. Examples are presented mainly in the soft $\mathrm{X}$-range allowing to probe the $3 d$ band of transition metals. They demonstrate the promising possibilities of the method to measure the magnetic moments carried by each of the atomic components in complex systems, as well as their distribution through thin layers, with an atomic resolution.
\end{abstract}

PACS numbers: 71.27.+a, 71.28.+d, 78.70.Ck

\section{Introduction}

In the past few years, X-ray resonant magnetic scattering (XRMS) has become one of the new useful techniques to analyse the magnetic properties of materials $[1,2]$. XRMS is a resonant magneto-optical Kerr effect (MOKE) in the X-ray range. It makes use of the magnetic contribution to the anomalous $\mathrm{X}$-ray scattering factor occurring right at an absorption edge. It is directly linked to X-ray magnetic circular dichroism (XMCD), which is nothing but its imaginary part. Therefore, XRMS offers the same advantages of chemical and orbital selectivity related to a resonant dipolar excitation from a core level at a well-defined photon energy. It may provide the same spectroscopic information as XMCD, on the magnetic state of an electronic shell. This selectivity is a major advantage in comparison with regular MOKE in the visible light or with neutron scattering. Being a scattering technique, it also allows probing the reciprocal space and thus yields information 
in real space on the magnetic structure in the sample. This is not possible using the MOKE method or the XMCD one.

An extensive work has already been performed on bulk materials using XRMS in the hard X-ray range, especially at the $L$ edges of rare earths' and at the $M$ edges of actinides [1]. For $3 d$ transition metals, which are the most important magnetic atoms, the only edge available in the hard X-ray range is the $K$ one. However, the corresponding magnetic resonance is very weak. On the contrary, strong effects have been observed in the soft X-ray range at their $L_{2}$ and $L_{3}$ edges, because the dipole transition directly probes the magnetic $d$ band. Such an enhancement has been first measured in the specular reflectivity pattern of a ferromagnetic $\mathrm{Fe}(110)$ thin film by Kao et al. [3], and later on in the diffraction spectrum of an $\mathrm{Ag} / \mathrm{Ni}$ multilayer at the $\mathrm{Ni} L$ edges [4]. As an example, Fig. 1 shows the diffraction pattern obtained at low angles for a $\mathrm{Co} / \mathrm{Cu}$ multilayer, at the $L_{2,3}$ edges of Co. Huge changes in the intensities of the two Bragg peaks are observed upon reversal of the magnetisation, with asymmetry ratios as large as $60 \%$ at the third order.

(a)

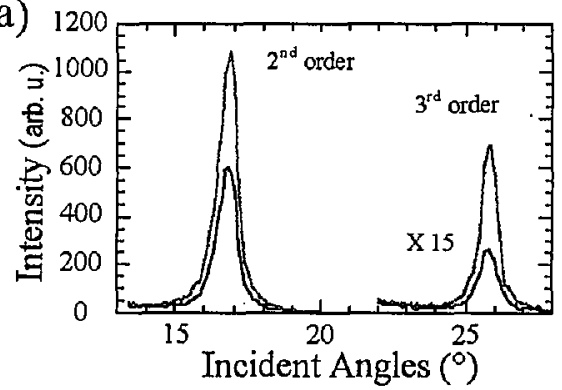

(b)

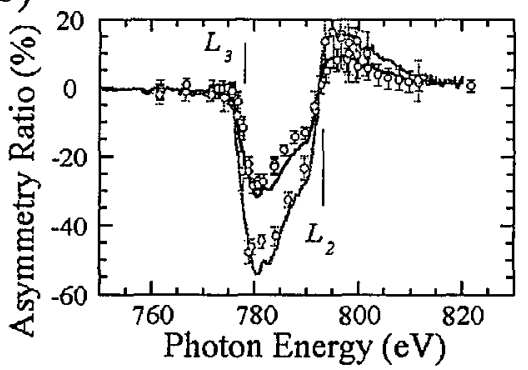

Fig. 1. (a) Second and third diffraction peaks measured at the Co $L_{3}$ edge for a Co/Cu multilayer. The two curves are obtained using a linearly polarised X-ray beam in a transverse geometry, by reversing magnetisation which is perpendicular to the scattering plane. (b) Experimental (dots) and calculated (full lines) energy dependencies of the asymmetry ratios over the $L_{2}$ and $L_{3}$ edges. The experiment has been performed using the U4B beam line, at the NSLS at Brookhaven.

In this paper, we only discuss the case of scattering at low values of the scattering vector $k$, either in reflectivity or diffraction modes. Such measurements are well suited to the investigation of the magnetic structure in thin films and artificial structures which often do not exhibit diffraction peaks at large angles because of a small coherence length. They can be performed in the hard X-ray range, but also in the soft X-ray one, since $k$ values of $1 \AA^{-1}$ can be reached with $1 \mathrm{keV}$ photons [4,5]. Despite the limited $k$ range, they may provide information on the magnetic structure with an atomic resolution [6]. Indeed, this is the scale of interest for most of the magnetic features of importance for magnetic thin films or artificial nanostructures, such as for instance the magnetic roughness at the interfaces, or the profile of the magnetisation across a thin layer. 


\section{The XRMS method}

XRMS makes use of the resonant enhancement of the magnetic scattering occurring at an absorption edge. For instance, in the case of the $L_{2(3)}$ edges in a transition metal, it results from the electric dipole transition from the $2 p_{1 / 2(3 / 2)}$ atomic core level towards the unoccupied $3 d_{3 / 2(5 / 2)}$ states which carry the magnetic moment. As for XMCD, the magnetic sensitivity arises from the exchange splitting of the unoccupied $3 d$ states induced by their magnetic polarisation, and from the spin polarisation of the photoelectron which is related to the spin-orbit coupling in the $2 p$ core level $[7,8]$. The imaginary part of the resonant magnetic scattering factor (MSF) is proportional to the XMCD amplitude. Neglecting the weak term related to the linear magnetic dichroism [7], the atomic scattering factor may be written as

$$
F(k, E)=-\left(e_{\mathrm{f}} \cdot e_{\mathrm{i}}\right) f(k, E)-\mathrm{i}\left(e_{\mathrm{f}} \cdot e_{\mathrm{i}}\right) z m(E)
$$

with

$$
f(k, E)=f_{0}(k)+f^{\prime}(E)-\mathrm{i} f^{\prime \prime}(E) \text { and } m(E)=m^{\prime}(E)-\mathrm{i} m^{\prime \prime}(E) .
$$

$\boldsymbol{e}_{\mathrm{i}}$ and $\boldsymbol{e}_{\mathrm{f}}$ are the polarisation vectors of the electric field for the incident and scattered X-ray beams. $E$ is the photon energy and $k$ - the scattering vector. $z$ is the unit vector along the direction of the magnetisation. The first term in (1) is the regular charge scattering by the electrons of the atom, including both the form factor $f_{0}(k)$ and the anomalous complex contribution associated to the absorption edge, $f^{\prime}(E)-\mathrm{i} f^{\prime \prime}(E)$. The second term in (1) is the resonant magnetic scattering factor. Its energy dependent complex amplitude depends on the spin and orbital magnetic moments through the $F_{1,1}+F_{1,-1}$ matrix element of the dipole transition $[7,8]$. Its real and imaginary parts, $m^{\prime}$ and $m^{\prime \prime}$, are linked by the Kramers-Kronig relation, as $f^{\prime}$ and $f^{\prime \prime}$ are.

The $m^{\prime \prime}(E)$ values are usually derived from the measurement of the XMCD amplitude as a function of energy, and the $m^{\prime}(E)$ ones are calculated using the Kramers-Kronig transformation. The $m^{\prime}$ and $m^{\prime \prime}$ values can also be derived from the simulation of the energy dependence of the XRMS intensity, provided that the magnetic structure of the sample is known [5]. One has to use an iterative process. At step $n, m_{n}^{\prime}(E)$ are extracted from the simulation of the XRMS data using the $m_{n-1}^{\prime \prime}$ set of values, then the $m_{n}^{\prime \prime}(E)$ data set is calculated using the Kramers-Kronig relationship, and the process is repeated until stable values are reached. As an example, Fig. 2 shows the $m^{\prime \prime}$ values determined in such a way for $\mathrm{Ni}$ at the $L_{2,3}$ edges, from the simulation of the energy dependence of the first diffraction peak from a Ni/Ag multilayer. They are compared with those directly measured from the XMCD amplitude for a thin film of pure Ni. An overall good agreement is observed, showing that the magnetisation state of $\mathrm{Ni}$ in $17.5 \AA$ thick layers is very similar to that in bulk Ni. This demonstrates that XRMS can be actually used as a spectroscopic technique, in the same way as XMCD. The quality of the determination of the MSF even makes possible the use of the sum rules over the $L_{2}$ and $L_{3}$ edges [8], which allows to separately determine the spin and orbital components in the magnetic moment. For instance, for $\mathrm{Ni}$ in an $\mathrm{Ni} / \mathrm{Ag}$ multilayer, their values were obtained with a good accuracy to be 


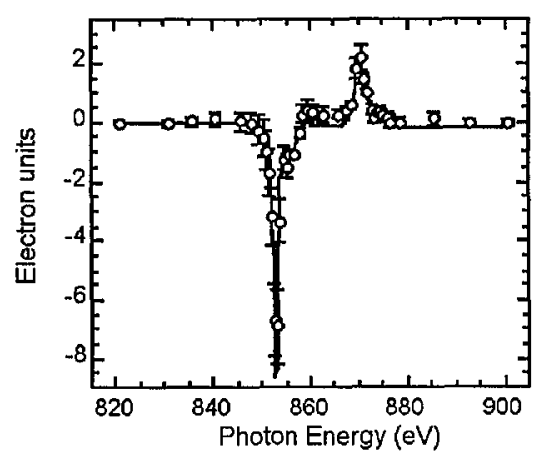

Fig. 2. Imaginary part of the resonant magnetic scattering factor of $\mathrm{Ni}$ at the $L_{2,3}$ edges determined from magnetic scattering for a $\mathrm{Ni} / \mathrm{Ag}$ multilayer (empty dots), compared to the magnetic dichroism in absorption for bulk $\mathrm{Ni}$ (full line). The experimental data were measured at LURE using a circularly polarised beam.

$0.37 \pm 0.06$ and $0.044 \pm 0.01 \mu_{\mathrm{B}}$, respectively [5]. The measurement of the orbital moment in thin films is of importance to understand their specific properties of magneto-anisotropy.

The angular dependence of the MSF is different from that of the charge one. As shown in Eq. (1), it depends on the projection of the cross product of the polarisation vectors along the direction of the magnetisation, so that the polarisation undergoes a rotation in the XRMS scattering. This introduces magnetic off diagonal terms in the dielectric tensor. Moreover, it allows the use of either linearly or circularly polarised X-rays, at variance with XMCD which needs a circular polarisation. Thus, as in regular MOKE experiments in the visible range, several geometries can be used in XRMS experiments, the longitudinal, polar and transversal ones. At weak scattering angles, which is usually the case in reflectivity measurements, the most efficient geometry is the longitudinal one using a circular polarisation of the X-ray beam, with the magnetisation in the plane of the layers and in the scattering plane. The transversal mode with the magnetisation perpendicular to the scattering plane and still in the plane of the layers, and the polar one with the magnetisation in the scattering plane but perpendicular to the plane of the layers, can both be used with a linear polarisation. They are more efficient at large incidence angles because of the dependence of the MSF amplitude on $\sin (2 \theta)$, with $\theta$ being the scattering angle.

\section{Magnetic structure of multilayers: diffraction at low $k$ values}

Due to their chemical modulation, multilayers with appropriate periods of a few nanometers are one-dimensional artificial crystals which allow Bragg diffraction at low scattering vectors using hard $\mathrm{X}$-rays, but also soft $\mathrm{X}$-ray photons with wavelengths in the nanometer range, the magnetic contribution being usually larger in that case. Such an example is displayed in Fig. 1. In comparison to reflectivity measurements, one major advantage of diffraction is the simplicity of the analysis. Even at low $k$ values, it is possible in most cases to use the kinematical 
theory which neglects multiple scattering. This is especially true in the soft X-ray range where the absorption length is far smaller than the extinction one, due to the huge absorption. For a multilayer, the intensity of a low angle Bragg peak is proportional to the square of the modulus of the structure factor $S(k, E)$. It is given by a summation over the unit cell with a height equal to the modulation period

$$
S^{ \pm}(k, E)=C(k, E) \pm M(k, E)=\sum[(f(k, E) \pm \mathrm{i} m(k)] \exp (\mathrm{i} k r) .
$$

The charge and magnetic contributions, $C$ and $M$, interfere with a sign dependent on the orientation of the magnetisation with respect to the X-ray beam polarisation. Thus the magnetic effect is better evidenced in the asymmetry ratio $R(k, E)$, defined as

$$
R(k, E)=\left(I^{+}-I^{-}\right) /\left(I^{+}+I^{-}\right) .
$$

The expression of $R$ depends on the scattering geometry and on the polarisation state of the beam. In the longitudinal mode using circular polarisation, it is proportional to $C_{\mathrm{r}} M_{\mathrm{r}}-C_{\mathrm{i}} M_{\mathrm{i}}[5]$, where $M_{\mathrm{r}}\left(C_{\mathrm{r}}\right)$ and $M_{\mathrm{i}}\left(C_{\mathrm{i}}\right)$ are the real and imaginary parts of $M(C)$, while it is proportional to $C_{\mathrm{r}} M_{\mathrm{i}}-C_{\mathrm{i}} M_{\mathrm{r}}$ in the transverse mode [9]. Its energy dependence thus may look like the real or the imaginary parts of the MSF, or like any mixture of them. This is illustrated in Fig. 3a and b, which show the asymmetry ratios in the longitudinal and transverse modes respectively, measured at the $L_{2,3}$ edges of $\mathrm{Ni}$ on the first Bragg peak for a $\mathrm{Fe}_{19} \mathrm{Ni}_{81} / \mathrm{Ag}$ multilayer. We point out that the $R$ amplitude and spectral shape strongly depend on the scattering angle. A good simulation thus implies an accurate knowledge of the structure of the sample.
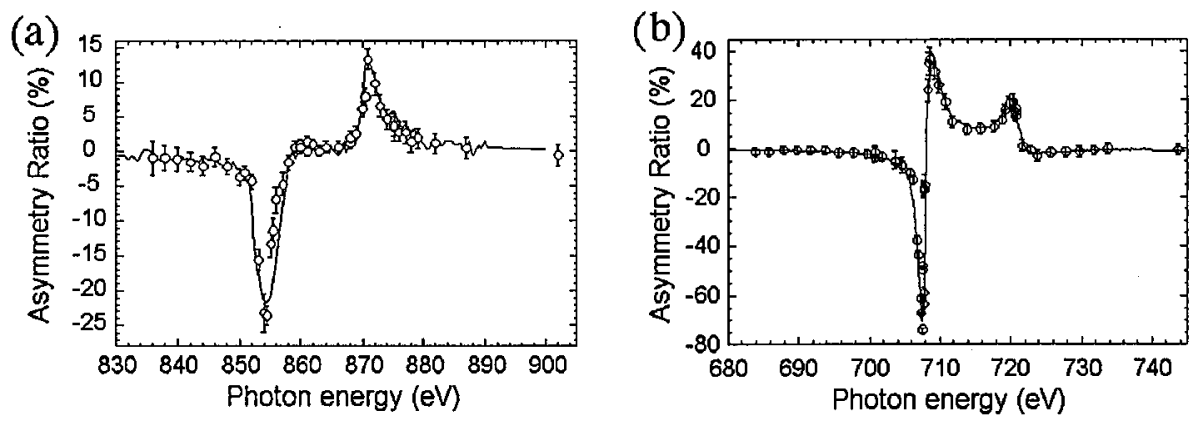

Fig. 3. Asymmetry ratios measured at the $L_{2,3}$ edges of $\mathrm{Ni}$ for a $\mathrm{Fe}_{19} \mathrm{Ni}_{81} / \mathrm{Ag}$ multilayer, on the first Bragg peak. The experiments have been performed at the U4B beam line at the NSLS at Brookhaven, in the (a) longitudinal and (b) transverse modes by using circularly and linearly polarised beams, respectively. Full lines are the calculations using the kinematical theory for diffraction.

\subsection{Permalloy and silver multilayers: a soft $X$-ray investigation}

These multilayered structures have been extensively studied by various techniques because of the large magnetoresistance which they exhibit for an appropriate thickness of the silver spacer layers inducing an antiferromagnetic coupling 
between the permalloy layers. Some of the physical properties of the thin permalloy layers in these nanostructures are different from those of bulk permalloy. For instance, neutron scattering experiments give a chemically averaged value of the magnetic moment of $0.86 \mu_{\mathrm{B}}$ at room temperature [10], which is significantly reduced in comparison with its $1 \mu_{\mathrm{B}}$ value in bulk permalloy. XRMS experiments have thus been performed at the $\mathrm{Fe}$ and $\mathrm{Ni} L$ edges to separately measure both moments. We would like to point out that XMCD measurements using total electron yield, which would also provide a separate evaluation of the $\mathrm{Ni}$ and $\mathrm{Fe}$ moments, could not be used for these samples because they were only sensitive to the oxidised top layers. The far larger depth probed in X-ray scattering turns out to be a significant advantage to investigate artificial structures which are not in situ prepared for the sake of the experiment.

The XRMS experiments have been performed both by registering the $1 / 2$ and $3 / 2$ peaks related to the antiferromagnetic coupling of the magnetic permalloy layers, and by measuring the asymmetry ratios for the regular modulation peaks. In the last case, a saturating magnetic field was applied to ferromagnetically couple the permalloy layers [11]. As an example, Fig. 3 shows the experimental values of the asymmetry ratio obtained in the last case, together with calculations obtained by using the $m^{\prime}$ and $m^{\prime \prime}$ values derived for bulk Ni from the XMCD amplitude. The structural parameters needed in the calculation of the structure factors $C$ and $M$ (thickness and atomic densities of the permalloy and $\mathrm{Ag}$ layers, roughness at the interfaces) were obtained from the simulation of the hard X-ray reflectivity spectrum. The only free parameter in the calculation is a scaling factor which allows to evaluate the magnetic moments in comparison with those in bulk $\mathrm{Ni}$ and Fe. For $13 \AA$ thick permalloy layers, values of the $\mathrm{Ni}$ and $\mathrm{Fe} 3 d$ magnetic moments of 0.57 and $2.07 \mu_{\mathrm{B}}$ were thus obtained at room temperature [11]. We first point out that the total moment $0.85 \mu_{\mathrm{B}}$ is in excellent agreement with the value derived from neutron scattering performed on the same sample [10]. The Ni magnetic moment is close to the bulk permalloy value $\left(0.6 \mu_{\mathrm{B}}\right)$. Unexpectedly however, the $\mathrm{Fe}$ one is significantly reduced in comparison with its $2.6 \mu_{\mathrm{B}}$ value in bulk permalloy. The origin of such a striking difference in the magnetic behaviours of $\mathrm{Fe}$ and $\mathrm{Ni}$ in thin layers is not yet clear. Simulations of the XRMS pattern show that it does not originate from a reduced Fe moment at the interfaces with $\mathrm{Ag}$ layers, since in such a case a similar decrease would also occur for the Ni moment, the homogeneity of the permalloy layers having been accurately checked by EXAFS measurements [11]. Experiments at low temperature, which should be feasible soon, are necessary to better understand the origin of the special behaviour of these thin layers. They nevertheless underline some of the possibilities of the method.

We present hereafter another example of application showing that XRMS also allows to measure the magnetic coupling energies between magnetic layers, which are important parameters to understand the magnetoresistance properties in multilayers exhibiting antiferromagnetic coupling between magnetic layers, as it is the case for $\mathrm{FeNi} / \mathrm{Ag}$. It deals with the strong dependence of the intensities of the antiferromagnetic Bragg peaks on the intensity $H$ of a magnetic field applied in the plane of the layers, below its saturation threshold. The magnetic interaction 
energy between to adjacent layers is given by

$$
E=2 J M^{2} \sin ^{2} \alpha+2 B M^{2} \sin ^{2}(2 \alpha)-H M t \cos \alpha,
$$

where $t$ and $M$ are the thickness and the magnetisation within a layer, respectively; $2 \alpha$ is the angle between the directions of magnetisation in two magnetic layers separated by a non-magnetic one. $J$ is the exchange coupling between them, which favours an antiparallel coupling, and $B$ is the biquadratic coupling, which may exist or not. The minimisation of $E$ gives an equation relating $\alpha$ to $H$ as a function of $J$ and $B$. This relation can actually be measured by XRMS using a linearly polarised incident X-ray beam with the polarisation vector in the scattering plane, since in such a case the intensity of the antiferromagnetic peaks depends on $\sin ^{2} \alpha$, allowing the measurement of the rotation angle $\alpha$. In turn, this allows to extract the coupling coefficients $J$ and $B$. In the case of $\mathrm{FeNi} / \mathrm{Ag}$ multilayers exhibiting a strong magnetoresistance, the results clearly show the existence of a significant biquadratic coupling, with values $J=-2.8 \times 10^{-6} \mathrm{~J} / \mathrm{m}^{2}$ and $B=-0.5 \times 10^{-6} \mathrm{~J} / \mathrm{m}^{2}$ [11]. This example is intended to show that XRMS offers the same possibilities as neutron scattering, with the advantage of chemical and orbital selectivities.

\subsection{Profiles of the magnetic polarisation in multilayers}

The angular dependence of the asymmetry ratios measured at several orders of diffraction for a multilayer turns out to be very sensitive to the magnetisation profile across thin magnetic layers and to the magnetic roughness at their interfaces with non-magnetic layers. For instance, in the case of the $\mathrm{Co} / \mathrm{Cu}$ sample the asymmetry ratios shown in the lower part in Fig. 1 can be simulated only by assuming that all Co atoms in the $37 \AA$ thick Co layers (19 atomic planes) do carry the same magnetic moment of $1.77 \mu_{\mathrm{B}}$, like in bulk Co. The simulations of the asymmetry ratios at the Co $L$ edges are very sensitive to the magnetic structure at the interfaces with $\mathrm{Cu}$ layers. For instance, a $50 \%$ reduction of the Co magnetic moment in the interfacial plane would even reverse the sign of the third-order one [11].

This sensitivity can be used to get the profile of magnetisation across layers, even in the case of weak moments such as those induced in non-magnetic layers by magnetic polarisation at their interfaces. This is of importance to understand the origin of the magnetic coupling between two magnetic layers through a non-magnetic one. As an example, we mention an extensive investigation of the magnetic profile of the $5 d[6]$ and $4 f$ [12] magnetic polarisation states of $\mathrm{Ce}$ in $\mathrm{Ce} / \mathrm{Fe}$ multilayers. It is well known that dramatic differences in the properties of Ce occur depending on its electronic and structural state. The situation is especially complex in the case of $\mathrm{Ce} / \mathrm{Fe}$ multilayers with thin Ce layers where $\mathrm{X}$-ray absorption spectroscopy indicates that $\mathrm{Ce}$ is in a $\alpha$-like state. However at variance with the regular $\alpha$ bulk phase appearing under pressure, XMCD experiments show that Ce is magnetically ordered at room temperature. Both the $5 d$ [13] and $4 f$ [14] Ce electronic states are magnetically polarised, the dependencies of the magnetisation upon the Ce layer thickness being different. The XRMS investigation at the Ce $M$ edge [12] supports the XMCD conclusion that the $4 f$ states are polarised 
by direct interaction at the interfaces. However, the $5 d$ states are found to be antiferromagnetically polarised, with the amplitude of the induced magnetic moments oscillating from a Ce atomic plane to the next one, and slowly decreasing from the interfaces with $\mathrm{Fe}$ towards the center of the Ce layer. Such an antiferromagnetic oscillating behaviour has been observed for several Ce thicknesses ranging from 10 to $25 \AA$, as well as for more complex structures, like $\mathrm{Fe} / \mathrm{Ce} / \mathrm{La} / \mathrm{Ce}$ ones. It could not be derived from XMCD measurements which yield the Ce moment averaged over the layers, which slowly decreases with their thickness.

\section{Magnetic resonant reflectivity}

Unfortunately, diffraction experiments often cannot be performed for artificial structures because of a limited coherence length preventing from getting coherent diffraction, even at low angles. Moreover, they cannot be performed using soft X-rays in the case of structures which do not exhibit a periodic modulation with the appropriate period. This is the case for thin films or structures with a small number of different layers. Reflectivity experiments at low scattering vector values are however always feasible providing useful information on their magnetic structure. In comparison with diffraction, the main disadvantage is the more difficult analysis, the treatment of reflectivity spectra implying the use of the optical dynamical theory taking account of full multiple scattering.

As an example, Figs. 4a and b show the measured and calculated angular dependencies of the reflectivity pattern and of the asymmetry ratio obtained by reversing the magnetisation measured at the $\mathrm{Fe} L_{3}$ edge for a $\mathrm{W}(32 \AA) / \mathrm{Fe}(91 \AA)$ / $\mathrm{W}(129 \AA)$ trilayer deposited by laser ablation on a $\mathrm{Al}_{2} \mathrm{O}_{3}$ substrate. The experiments were performed using the transversal mode with a linearly polarised X-ray beam. The angular dependencies exhibit many short period structures related to the Kiessing fringes, which are well reproduced in the calculation.

To analyse the data, we have developed a numerical calculation based on the optical approach where the Maxwell equations are solved in a matrix formalism. The main part is to calculate the dielectric tensor. The magnetic effects are introduced as off diagonal terms which are proportional to the complex MSF. Four waves do propagate in each layer along two directions, the downwards incident and upwards reflected ones, and with two orthogonal linear or circular polarisation states. A four dimension vector is thus used to describe the amplitude $E_{n}$ of the electric fields in layer $n$. Its value in layer $n-1$ is related to that in layer $n$ by a matrix equation

$$
E_{n-1}=\left(A_{n-1}\right)^{-1} A_{n} P_{n} E_{n} .
$$

The four by four matrices $A_{n}$ and $P_{n}$ are the medium boundary matrix allowing to write the boundary conditions for the fields at the interface between layers $n-1$ and $n$, and the diagonal propagation matrix through layer $n$, respectively. In the transversal geometry using linear polarisation which is the one shown in Fig. 4, the two $\sigma$ and $\pi$ polarisation states are not coupled, which makes their calculation easier $[11,15]$. This is not true in the general case, and especially in the longitudinal geometry using circular polarisation. Moreover in that case, 

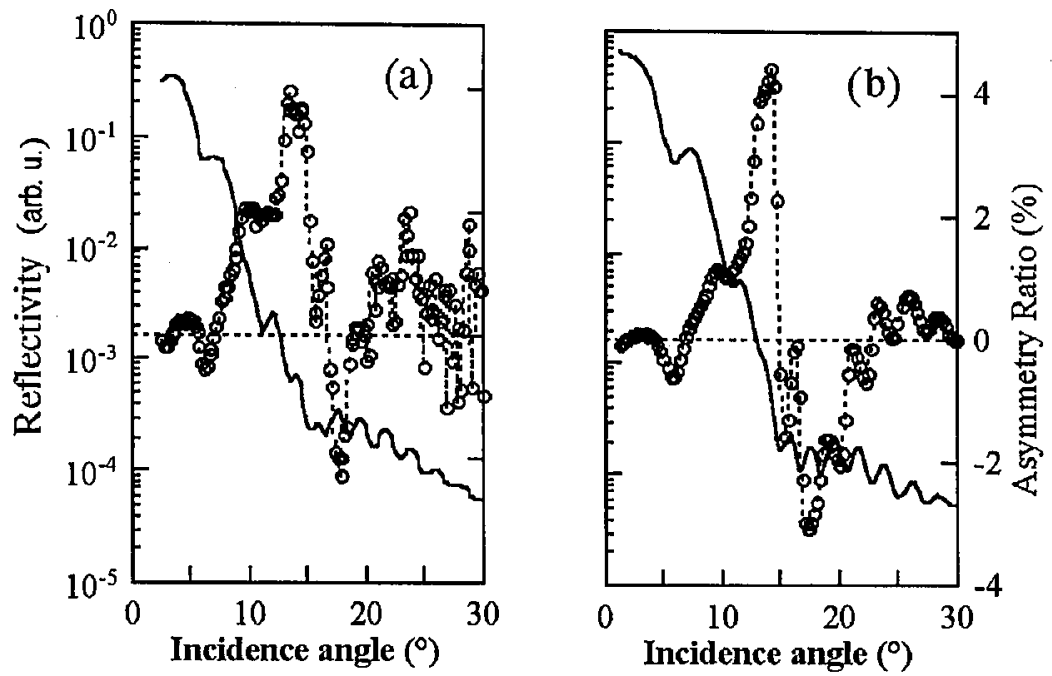

Fig. 4. (a) Measured and (b) calculated angular dependencies of the reflectivity pattern (full line) and of the asymmetry ratio (empty dots) obtained by reversing the magnetisation, measured at the $\mathrm{Fe} L_{3}$ edge for a W(32 $\left.\AA\right) / \mathrm{Fe}(91 \AA) / \mathrm{W}(129 \AA)$ trilayer deposited by laser ablation on a $\mathrm{Al}_{2} \mathrm{O}_{3}$ substrate. The experiments were performed on $\mathrm{U} 4 \mathrm{~B}$ at NSLS, using the transversal mode with a linearly polarised X-ray beam.

the right and left handed polarisation states experience a different attenuation resulting in a change in the rate of circular polarisation across the layer. This makes the calculation of the reflected intensity more difficult than for a linearly polarised beam since the polarisation state of the light reflected at the interface with the substrate is not a priori known. It is then necessary to calculate what would be the X-ray electric field incident on the sample, in the two cases of a right or left handed fully circular beam reflected from the substrate. Their amplitudes are then added with respective weights in order to reproduce the polarisation state of the incident beam, allowing then the calculation of the reflectivity [16]. The simulation shown in Fig. 5 has been done in such a way. The good agreement with experimental data validates the method.

Actually, we have found that $\mathrm{X}$-ray magnetic reflectivity is very sensitive to roughness at the interfaces between layers [15]. Thus, the optical formalism derived from the MOKE one has to be modified following the prescription of Vidal and Vincent [17]. In the Fresnel matrix $F=A_{n-1} A_{n} / T$, where $T$ is the transmission coefficient at the interface, the elements connecting the incident and reflected waves are weighted by a Debye-Waller like factor $e^{+}$, and those connecting incident waves (or reflected ones) by $e^{-}$, with

$$
e^{+}=\exp \left[-\left(k_{n}-k_{n-1}\right)^{2} \sigma^{2} / 2\right] \text { and } e^{-}=\exp \left[-\left(k_{n}+k_{n-1}\right)^{2} \sigma^{2} / 2\right] \text {. }
$$

$\sigma$ is the roughness parameter defined as the standard deviation in the distribution profile at the interface. The simulations shown in Fig. 4 and 5 have been calculated using such a formalism. They allow to fit the $\sigma$ values with a good accuracy. Actually, in this example, the simulation of the asymmetry ratio gives, for the 

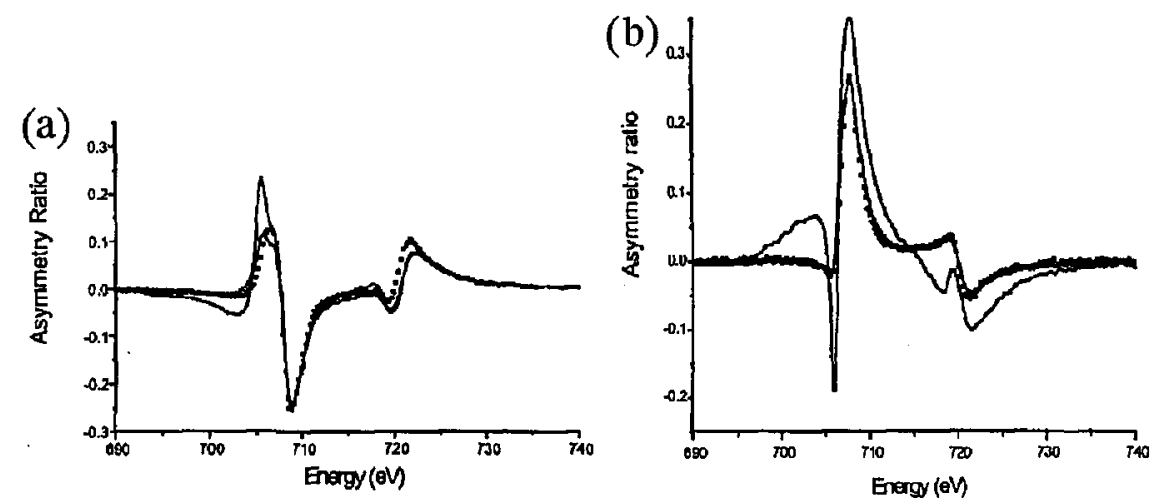

Fig. 5. Energy dependence of the asymmetry ratio at the $F e L_{2,3}$ edges for a $\mathrm{W}(32 \AA) / \mathrm{Fe}(91 \AA) / \mathrm{W}(129 \AA)$ trilayer, in the longitudinal mode using a circularly polarised beam, at two incidence angles (a) $10^{\circ}$, (b) $15^{\circ}$. Dots show experimental data, and full lines the simulations calculated with or without taking into account the roughness at the interfaces.

various interfaces, the same $\sigma$ values as those obtained from the fit of the total reflectivity using hard X-rays, suggesting that the magnetic roughness is only due to chemical disorder at the interfaces. We point out that only one $\sigma$ parameter can be introduced for each interface in our calculation. Thus, the chemical roughness that is due to the distribution of positions of the atoms at the interfaces, and the magnetic one that is due to the distribution of the projections of the magnetic moments on the polarisation vector of the electric field, cannot be independently evaluated. In principle, this limitation could be bypassed by explicitly describing the structure of the interface by slicing its width with an atomic step, as it has been done using the kinematical theory in the case of $\mathrm{Ce}(\mathrm{La}) / \mathrm{Fe}$ multilayers (see Sec. 3). To our knowledge, this has not yet been tried.

Despite this limitation, the technique is however already sufficiently mature to be efficiently used. As an example, we briefly mention an investigation of the magnetic properties of thin strained FeMn layers epitaxially deposited on a Ir (001) substrate with the goal of stabilising new phases with expanded volume and enhanced magnetic moment. Among $3 d$-transition metals, $\mathrm{Mn}$ is one of the most exciting since the Hund rules lead to a magnetic moment of $5 \mu_{\mathrm{B}}$. Bulk FeMn alloys are antiferromagnetic up to a $90 \% \mathrm{Fe}$ concentration. However, strained $\mathrm{Fe}_{x} \mathrm{Mn}_{1-x}$ thin films exhibit a structural and magnetic phase transition at $x=0.75$ [18]. SQUID magnetometry measurements suggest a transition from a zero net magnetic moment to a ferromagnetic one, without allowing its accurate measurement. This has prompted an investigation by magnetic resonant reflectivity at the $\mathrm{Fe}$ and $\mathrm{Mn} L$ edges, together with a detailed structural study as a function of the Mn concentration [19]. Indeed, the results show that both states on each side of the transition at $x=0.75$ are ferromagnetic, at variance with bulk results. The transition occurs between a low spin state with a magnetic moment of $0.27 \mu_{\mathrm{B}}$ per Fe atom to a high spin one with $2.1 \mu_{\mathrm{B}}$. Mn atoms are also found to be ferromagnetically ordered, but are antiferromagnetically coupled to the Fe ones, with a 
magnetic moment reaching up to $1.7 \mu_{\mathrm{B}}$ in Fe rich samples. This antiferromagnetic coupling is opposite to most of the available experimental results on Mn diluted in Fe bulk samples. Actually, the investigation of samples at various compositions, with different strain states, has clarified the origin of the special magnetic behaviour of the thin films in comparison with bulk alloys. The main parameter is not the atomic volume, but the $c / a$ distortion of the tetragonal centred structure. The transition from a low to high spin state occurs at the critical value 1.2 which also corresponds to a structural transition between fcc- and bcc-like structures.

\section{Conclusions}

$\mathrm{X}$-ray resonant magnetic scattering at low values of the scattering vector is a new technique, still under development. Reflectivity measurements are especially well suited to the investigation of magnetic thin films and artificial structures, though diffraction can also be used for multilayered systems. XRMS offers the advantages of XMCD and of neutron scattering at low angles simultaneously, since it allows to determine the magnetic structure in such samples with a chemical selectivity. It even allows to probe independently the magnetic polarisation of each electronic shell in a magnetic atom. This is of importance to understand the magnetic properties of complex materials. We have shown that the method can be used as a spectroscopic tool as for XMCD, in order to measure the magnetic moments of each component in complex materials, but also as a structural tool allowing to get information on the magnetic ordering in thin layers with an atomic resolution. The technique turns out to be very sensitive to the structure of interfaces.

\section{References}

[1] D.B. Mac Whan, J. Synch. Rad. 1, 83 (1994) and references therein.

[2] J.M. Tonnerre, in: Magnetism and Synchrotron Radiation, Eds. E. Beaurepaire, B. Carrière, J.P. Kappler, Les Editions de Physique, Paris 1997, p. 245 and references therein.

[3] C.C. Kao, J.B. Hastings, E.D. Johnson, D.P. Siddons, G.C. Smith, G.A. Prinz, Phys. Rev. Lett. 65, 373 (1990).

[4] J.M. Tonnerre, M. Jergel, D. Raoux, M. Idir, G. Soulier, R. Barchewitz, B. Rodmaq, J. Magn. Magn. Mater. 121, 230 (1993).

[5] J.M. Tonnerre, L. Séve, D. Raoux, B. Rodmacq, G. Soullié, P. Wolfers, Phys. Rev. Lett. 75, 740 (1995).

[6] L. Séve, N. Jaouen, J.M. Tonnerre, D. Raoux, F. Bartolomé, M. Arend, W. Felsch, A. Rogalev, J. Goulon, C. Gautier, J.F. Bérar, Phys. Rev. B 60, 9662 (1999).

[7] J.P. Hannon, G.T. Trammell, M. Blume, D. Gibbs, Phys. Rev. Lett. 61, 1245 (1988).

[8] P. Carra, M. Altarelli, Phys. Rev. Lett. 64, 1286 (1990).

[9] J.M. Tonnerre, L. Sève, D. Raoux, B. Rodmaq, M. de Santis, P. Troussel, V. Chakarian, C.C. Kao, E. Johnson, C.T. Chen, Nucl. Instrum. Methods Phys. Res. B 97, 444 (1995). 
[10] B. Rodmacq, K. Dumesnil, P. Mangin, M. Hennion, Phys. Rev. B 48, 3556 (1993).

[11] L. Sève, Ph. D. thesis, Université J. Fourier, Grenoble 1997.

[12] L. Sève, N. Jaouen, J.M. Tonnerre, D. Raoux, M. Arend, W. Felsch, F. Bartolomé, A. Rogalev, J. Goulon, V. Chakarian, Jpn. J. Appl. Phys. 38, 385 (1999).

[13] M. Arend, M. Finazzi, O. Schulte, M. Münzenberg, A.M. Dias, F. Baudelet, C. Giorgetti, E. Dartyge, P. Schaaf, J.P. Kappler, W. Felsch, G. Krill, Phys. Rev. B 57, 2174 (1998).

[14] M. Finazzi, F.M.F. de Groot, A.M. Dias, B. Kierren, F. Bertran, Ph. Sainctavit, J.P. Kappler, O. Schulte, W. Felsch, G. Krill, Phys. Rev. Lett. 75, 4654 (1995).

[15] J. M. Tonnerre, L. Sève, A. Barbara, F. Bartolomé, D. Raoux, V. Chakarian, C.C. Kao, H. Fischer, S. Andrieu, O. Fruchart, J. Appl. Phys. 83, 6293 (1998).

[16] E. Bontempi, J.M. Tonnerre, submitted for publication.

[17] B. Vidal, P. Vincent, J. Appl. Opt. 8, 1794 (1984).

[18] S. Andrieu, H. Fischer, A. Traverse, M. Piecuch, Phys. Rev. B 54, 2822 (1996).

[19] A. Dechelette, J.M. Tonnerre, M.C. Saint-Lager, F. Bartolomé, L. Sève, D. Raoux, H. Fischer, M. Piecuch, V. Chakarian, C.C. Kao, Phys. Rev. B 60, 6636 (1999). 